\title{
MODEL-BASED FAULT DETECTION AND IDENTIFICATION WITH ONLINE AERODYNAMIC MODEL STRUCTURE SELECTION
}

\section{T. Lombaerts}

German Aerospace Center DLR, Insititute of Robotics and Mechatronics 20 Münchner Straße, Weßling-Oberpfaffenhofen 82234, Germany

Delft University of Technology, Faculty of Aerospace Engineering 1 Kluyverweg, HS Delft 2629, the Netherlands

\begin{abstract}
This publication describes a recursive algorithm for the approximation of time-varying nonlinear aerodynamic models by means of a joint adaptive selection of the model structure and parameter estimation. This procedure is called adaptive recursive orthogonal least squares (AROLS) and is an extension and modification of the previously developed ROLS procedure. This algorithm is particularly useful for model-based fault detection and identification (FDI) of aerospace systems. After the failure, a completely new aerodynamic model can be elaborated recursively with respect to structure as well as parameter values. The performance of the identification algorithm is demonstrated on a simulation data set.
\end{abstract}

\section{INTRODUCTION}

Fault detection and isolation is a crucial control engineering aspect to guarantee the safe operation of complex safety-critical systems, such as nuclear powerplants and transport aircraft. An important FDI category is model-based FDI, where a mathematical model is relied upon to detect the occurrence of a fault. Parameter identification provides an important contribution in the elaboration of this mathematical model. This publication will focus on parameter identification for model-based FDI, inclined to aeronautics applications.

A number of aerodynamic model identification methods in the context of faults/failures have been proposed in the literature, like the use of indicial functions for unsteady aerodynamic effects [1], real-time identification in the frequency domain $[2,3]$, and modified sequential least squares [4]. These publications demonstrate specific advantages for each identification method. However, 
for these online methods, the conventional model structure is usually considered fixed and the aerodynamic derivatives are deduced by means of some recursive or sequential least squares procedure. However, this conventional structure may be especially invalid during highly dynamic manoeuvres and in damaged situations. In these settings, it is highly probable that the conventional linear aircraft model structure must be extended with additional nonlinear and/or coupling terms and their accompanying derivatives. In this kind of applications, one is not certain which independent variables may or may not have a significant influence on the dependent variable, which makes it important to apply some measure of structure selection. There are some good reasons for this. One of them is the principle of parsimony.

Definition 1. Principle of parsimony: if there are two mathematical models to represent the same system with equal accuracy, then the model with the fewest parameters is preferable.

This principle promotes computational speed, which is especially important for online applications like here. Another fact is that including many insignificant data in the regressor set will lead to many small coefficients with large standard deviations due to ill-conditioning. Besides, these irrelevant estimates, although small but numerous, result in biased estimates of the coefficients for the significant regressors.

Suppose the true system can be described as: $\mathbf{z}=\boldsymbol{\Phi}_{\text {sign }} \cdot \boldsymbol{\theta}$. Due to a lack of knowledge about the model structure, its approximation is defined as follows:

$$
\mathbf{z}=\boldsymbol{\Phi}_{\text {sign }} \hat{\boldsymbol{\theta}}_{\text {sign }}+\boldsymbol{\Phi}_{\text {insign }} \hat{\boldsymbol{\theta}}_{\text {insign }}
$$

where $\boldsymbol{\Phi}_{\text {sign }}$ contains the significant regressors and $\boldsymbol{\Phi}_{\text {insign }}$ is the collection of insignificant regressors. Premultiplying (1) with $\left(\boldsymbol{\Phi}_{\text {sign }}^{\mathrm{T}} \boldsymbol{\Phi}_{\text {sign }}\right)^{-1} \boldsymbol{\Phi}_{\text {sign }}^{\mathrm{T}}$ results in

$$
\hat{\boldsymbol{\theta}}_{\text {sign }}=\boldsymbol{\theta}-\left(\boldsymbol{\Phi}_{\text {sign }}^{\mathrm{T}} \boldsymbol{\Phi}_{\text {sign }}\right)^{-1} \boldsymbol{\Phi}_{\text {sign }}^{\mathrm{T}} \boldsymbol{\Phi}_{\text {insign }} \hat{\boldsymbol{\theta}}_{\text {insign }}
$$

which indicates a discrepancy between the parameter estimate $\hat{\boldsymbol{\theta}}_{\text {sign }}$ and the true parameter $\boldsymbol{\theta}$. This deviation becomes larger for $\hat{\boldsymbol{\theta}}_{\text {insign }}$ with larger size and if $\boldsymbol{\Phi}_{\text {sign }}$ and $\boldsymbol{\Phi}_{\text {insign }}$ are spanned in space by mutual linear dependent bases and if $\boldsymbol{\Phi}_{\text {sign }}$ is not well-defined. There is no deviation if $\boldsymbol{\Phi}_{\text {sign }}$ and $\boldsymbol{\Phi}_{\text {insign }}$ are mutually orthogonal, which is generally not the case.

Therefore, proper identification, including structure selection, is not avoidable for a damaged aircraft with changed aerodynamic properties. In this paper, the aim of this routine is to perform adaptive structure selection and parameter estimation for an aerodynamic model of a structurally damaged aircraft. This allows to exploit the knowledge of these data for a model-based FDI technique. In this way, safety and survivability will be enhanced. Earlier results of identification and fault tolerant flight control investigations in this research project have 
been published previously [5-9]. This earlier work focuses on fixed model structures and offline structure selection. This paper highlights a new development, namely, online structure selection.

There are many interesting structure selection and parameter estimation (SSPE) algorithms, like stepwise regression $[10,11]$. It is a very physical and intuitive procedure, but beside being not (yet) recursive, its main drawback is that it includes addition and elimination criteria. Therefore, it is less efficient than OLS that involve only a forward selection procedure. Especially for online applications, this is an important advantage. Orthogonal least squares have been used before for nonlinear modeling and roll derivatives estimation from flight data [12]. However, they have only been applied for batch data and not for damaged aircraft. For nonlinear aerodynamic modeling problems, the similar idea of generating multivariate orthogonal modeling functions from measured data, ranking those orthogonal functions by fit error reduction capability, and using the predicted square error (PSE) metric for model structure determination was originally developed by Morelli [13-16]. The PSE metric was originally developed earlier by Barron [17]. Moreover, orthogonal functions were used earlier by Mulder [18] in the optimization of multidimensional input signals for dynamic flight test maneuvers. However, this paper focuses on a new alternative approach via the working principle of OLS, which is computationally efficient for recursive applications.

The concept of ROLS has been introduced in [19-22], where the concept of structure selection has been highlighted. This is a very interesting and powerful routine, which can also be applied for damaged aircraft model identification, as explained above. However, for this aerospace application, some modifications are needed in the routine. Especially for online applications, it is important to include some protection against overfitting for computational speed and robustness considerations, which can be done by choosing an appropriate monitoring variable in the routine. Besides, in damaged situations, the physical system model changes abruptly and the routine must be rendered adaptive in order to deal with this varying structure. The objective of this paper is to solve these problems and to elaborate a basic routine which is demonstrated for aerospace applications.

\section{THE PRINCIPLE OF ADAPTIVE RECURSIVE ORTHOGONAL LEAST SQUARES}

Recursive OLS are very similar to the classical OLS structure, but the procedure is rewritten in order to take into account a stepwise growing data matrix and vector of the dependent variable. The SSPE by ROLS has been mentioned earlier by Luo et al. [20]. This model structure selection and parameter estimation 
algorithm is based on orthogonal decompositions [23] and involves Givens rotations to be used for recursive orthogonal transformations, retriangularization and online structure detection [20]. The AROLS algorithm is an extension to ROLS. Specifically, a last step has been added to make the routine adaptable for changes in the dynamics of the true system. The advantages of AROLS are also illustrated in the application example shown in section 3 .

This recursive procedure is based upon the property that the columns in the $\mathbf{R}$ matrix of a $\mathbf{Q R}$ decomposition can be interchanged multiply and arbitrarily, as long as the product is compensated backwards by a proper permutation matrix.

Suppose that $\mathbf{\Phi}=\mathbf{Q R}$ and define $\mathbf{R}^{(1)}=\mathbf{R \Pi}_{1}$, where permutation matrix $\boldsymbol{\Pi}_{1}$ is orthonormal; so, $\boldsymbol{\Pi}_{1}^{-1}=\boldsymbol{\Pi}_{1}^{\mathrm{T}}$. Correspondingly,

$$
\mathbf{R}=\mathbf{R}^{(1)} \boldsymbol{\Pi}_{1}^{-1}=\mathbf{R}^{(1)} \boldsymbol{\Pi}_{1}^{\mathrm{T}} .
$$

Since $\mathbf{R}^{(1)}$ is not purely upper triangular, it can be decomposed as

$$
\mathbf{R}^{(1)}=\mathbf{Q}_{N} \mathbf{R}_{N}^{(1)} .
$$

Substituting all this for the original data matrix $\boldsymbol{\Phi}$ results in

$$
\boldsymbol{\Phi}=\mathbf{Q R}^{(1)} \boldsymbol{\Pi}_{1}^{\mathrm{T}}=\underbrace{\mathbf{Q} \mathbf{Q}_{N}}_{\mathbf{Q}^{(1)}} \mathbf{R}_{N}^{(1)} \boldsymbol{\Pi}_{1}^{\mathrm{T}} .
$$

Now define the second permutation:

$$
\mathbf{R}_{N}^{(2)}=\mathbf{R}_{N}^{(1)} \boldsymbol{\Pi}_{2}
$$

Correspondingly,

$$
\mathbf{R}_{N}^{(1)}=\mathbf{R}_{N}^{(2)} \boldsymbol{\Pi}_{2}^{\mathrm{T}}
$$

Since $\mathbf{R}_{N}^{(2)}$ is not purely upper triangular, it can once again be decomposed as

$$
\mathbf{R}_{N}^{(2)}=\mathbf{Q}_{2 N} \mathbf{R}_{2 N}^{(2)}
$$

Substituting all this for the original data matrix $\boldsymbol{\Phi}$ results in

$$
\boldsymbol{\Phi}=\underbrace{\mathbf{Q}^{(1)} \mathbf{Q}_{2 N}}_{\mathbf{Q}^{(2)}} \mathbf{R}_{2 N}^{(2)} \underbrace{\boldsymbol{\Pi}_{2}^{\mathrm{T}} \boldsymbol{\Pi}_{1}^{\mathrm{T}}}_{\boldsymbol{\Pi}_{\text {tot }}^{\mathrm{T}}} .
$$

The principle holds by recursion for more permutations.

A visual overview of the procedure can be found in Fig. 1, where a clear distinction is made between the 'standard' steps to be performed under any circumstance, on the left, and the 'optional' steps on the right, of which the 


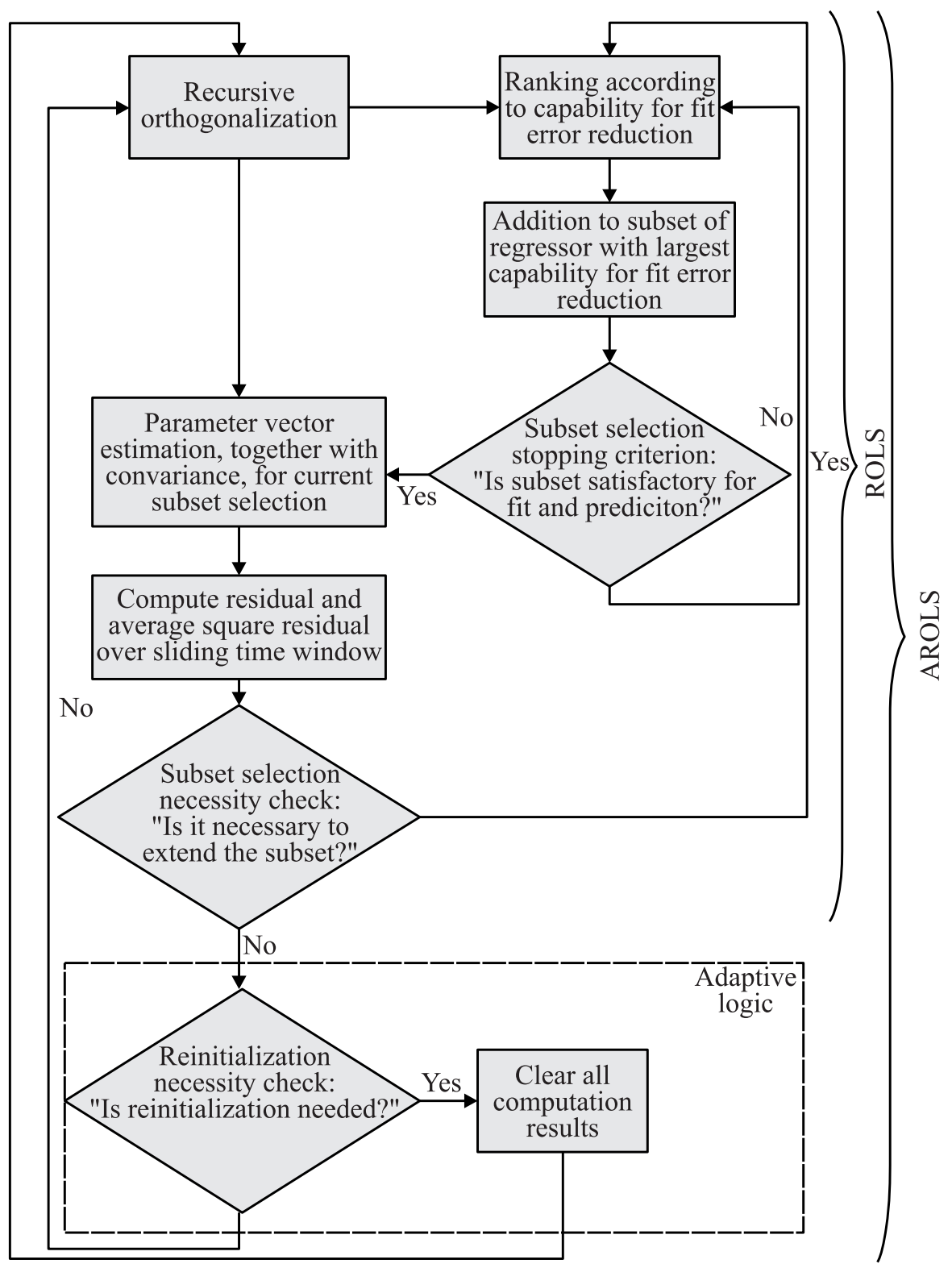

Figure 1 Overview of the steps of the AROLS procedure 
execution is triggered when needed. The detailed procedure of this algorithm is split up between an initialization phase and a recursive phase to be repeated for every time step.

\section{INITIALIZATION}

Generally, the $\mathbf{Q R}$ decomposition works as follows:

$\mathbf{z}(t)=\boldsymbol{\Phi}(t) \boldsymbol{\theta}(t)+\boldsymbol{\nu}(t)$.

$\boldsymbol{\Phi}(t)=\mathbf{Q}(t) \mathbf{R}(t)$ with $\mathbf{Q}^{\mathrm{T}}(t) \mathbf{Q}(t)=\mathbf{I}$ with $\mathbf{I}$ being the identity matrix and $\mathbf{R}(t)$ being the upper triangular.

Calculate: $\mathbf{v}(t)=\mathbf{Q}^{\mathrm{T}}(t) \mathbf{z}(t)$ since $\mathbf{v}(t)=\mathbf{Q}^{\mathrm{T}}(t) \mathbf{z}(t)=\mathbf{R}(t) \boldsymbol{\theta}(t)+\mathbf{Q}^{\mathrm{T}}(t) \boldsymbol{\nu}(t)$.

As initialization, the following initial values can be defined: $\mathbf{R}(t-1)=\mathbf{I}_{m}$ and $\mathbf{v}_{m}(t-1)=\mathbf{0}_{m \times 1}$.

Construct the augmented matrix:

$\mathbf{R}_{\text {aug }}(t-1)=\left[\begin{array}{cc}\mathbf{R}(t-1) & \mathbf{v}_{m}(t-1) \\ \mathbf{0}_{1} & \mathbf{0}_{2}\end{array}\right]$.

$\boldsymbol{\Pi}_{0}=\mathbf{I}_{m+1}$

\section{TO BE REPEATED FOR EVERY TIME STEP}

1. Multiply this augmented matrix with forgetting factor $\lambda^{1 / 2}$ and put the new data in a new row:

$$
\mathbf{R}_{\mathrm{aug}}(t)=\left[\begin{array}{c}
{\left[\lambda^{1 / 2} \mathbf{R}(t-1) \lambda^{1 / 2} \mathbf{v}_{m}(t-1)\right]} \\
{\left[\phi_{1}(t), \ldots, \phi_{m}(t) z(t)\right] \mathbf{\Pi}_{\mathrm{tot}}}
\end{array}\right] .
$$

2. Using Givens rotations produce the new augmented matrix:

$$
\mathbf{R}_{\text {aug }_{\text {new }}}(t)=\left[\begin{array}{cc}
\mathbf{R}(t) & \mathbf{v}_{m}(t) \\
\mathbf{0}_{1} & \mathbf{0}_{2}
\end{array}\right] .
$$

3. When the $j$ th regressor is being selected, compute $v_{m(p)}^{2}(t), p=j, \ldots, m$, $j \geq 1$, based upon the updated values $\mathbf{v}_{m}(t)$ in Eq. (2) and choose the $k$ th optimal regressor with the maximum $v_{m(p)}^{2}(t), p=j, \ldots, m$, for the $j$ th position by earmarking the appropriate column of $\mathbf{R}(t)$.

4. This step can be skipped in the case where $j=k$.

According to the result of the previous step, as the $k$ th variable has been selected, exchange the positions of the current $j$ th and $k$ th columns of $\mathbf{R}(t)$ and then retriangularize $\mathbf{R}(t)$ and rotate $\mathbf{v}_{m}(t)$ via $\mathbf{Q R} .{ }^{*}$ Mind that $j \leq k$.

${ }^{*}$ Mind that calculating $\mathbf{Q}$ is not necessary here. All required information is contained in the upper triangular $\mathbf{R}$. This saves a considerable amount of computational load, since especially the size of $\mathbf{Q}$ increases with the number of data samples. 
Define permutation matrix $\boldsymbol{\Pi}_{j k}$ accordingly:

$$
\boldsymbol{\Pi}_{j k}=\left[\begin{array}{ccccc}
\mathbf{I}_{j-1, j-1} & 0 & 0 & 0 & \mathbf{0}_{j-1, m-k+1} \\
0 & 0_{j, j} & 0 & 1_{j, k} & 0 \\
0 & 0 & \mathbf{I}_{k-j, k-j} & 0 & 0 \\
0 & 1_{k, j} & 0 & 0_{k, k} & 0 \\
\mathbf{0}_{m-k+1, j-1} & 0 & 0 & 0 & \mathbf{I}_{m-k+1, m-k+1}
\end{array}\right]
$$

and compute the total permutation matrix as the product of all individual permutation matrices $\boldsymbol{\Pi}_{\text {tot }}=\prod\left(\boldsymbol{\Pi}_{j k}\right)$

5. Evaluate a subset selection stopping criterion and perform the next step if this condition is satisfied; otherwise, return to step 3 to select more regressors. There are some alternatives for this subset selection stopping criterion, and this subject is elaborated further in a later stage.

6. Suppose that $m_{s}$ regressors have been selected, then the computational augmented matrix is:

$$
\mathbf{R}_{\text {aug }}(t)=\left[\begin{array}{ccccccc}
r_{11}(t) & \cdots & \cdots & r_{1, m_{s}}(t) & \cdots & r_{1, m-1}(t) & v_{1}(t) \\
0 & \ddots & \vdots & \vdots & \cdots & \vdots & \vdots \\
\vdots & \ddots & \ddots & \vdots & \cdots & \vdots & \vdots \\
\vdots & \cdots & 0 & r_{m_{s}, m_{s}}(t) & \ddots & \vdots & v_{m_{s}}(t) \\
\vdots & \cdots & 0 & \ddots & \vdots & \vdots \\
\vdots & \vdots & \vdots & \vdots & \ddots & r_{m-1, m-1}(t) & v_{m}(t) \\
0 & \cdots & \cdots & 0 & \cdots & 0 & 0
\end{array}\right] .
$$

7. Using back-substitution, solve for the parameters $\hat{\theta}_{i}(t), i=1, \ldots, m_{s}$, from $\mathbf{R}_{m_{s}}(t)$, which is the top-left triangular portion of the final $\mathbf{R}_{\text {aug }}(t)$, and $\mathbf{v}_{m_{s}}(t)$ which consists of the first $m_{s}$ elements of $\mathbf{v}_{m}(t)$. The standard deviation can be calculated by exploiting the property that $\left(\mathbf{\Phi}_{s}^{\mathrm{T}} \mathbf{\Phi}_{s}\right)^{-1}$ $=\left(\mathbf{R}_{m_{s}}^{\mathrm{T}} \mathbf{Q}_{s}^{\mathrm{T}} \mathbf{Q}_{s} \mathbf{R}_{m_{s}}\right)^{-1}=\left(\mathbf{R}_{m_{s}}^{\mathrm{T}} \mathbf{R}_{m_{s}}\right)^{-1}$

8. Compute the residual at the time instant $t, \boldsymbol{\varepsilon}(t)=\mathbf{z}(t)-\sum_{i=1}^{m_{s}} \boldsymbol{\phi}_{i}(t) \hat{\theta}_{i}(t)$. This result is used to calculate the average square residual in the sliding time window $M_{s}: \overline{\varepsilon_{M_{s}}^{2}}(t)=\left(1 / M_{s}\right) \sum_{i=0}^{M_{s}-1} \varepsilon^{2}(t-i)$, with typically $M_{s}$ 
$=20-100$ (choice here: 50). If the average square residual is below the predefined threshold $\overline{\varepsilon_{M_{s}}^{2}}(t) \leq \xi_{M_{s_{1}}}=0.2$, then steps 4 to 6 can be omitted and $m_{s}$ remains unchanged. If average residual exceeds predefined threshold, steps 4 to 6 need to be performed again.

9. If average square residual exceeds the second, more relaxed threshold $\overline{\varepsilon_{M_{s}}^{2}}(t) \geq \xi_{M_{s_{2}}}=0.3$ and if the standard deviation of all coefficients is below a predefined third threshold $\sigma_{j}<\xi_{M_{s_{3}}}{ }^{*}$, then all previous measurement data can be ignored and the procedure has to start over again. The motivation for this is the fact that for higher values of standard deviations, structure selection is most appropriate. If the standard deviations are low and the average square residual exceeds a predefined threshold, one obtains an indication that the true system dynamics have changed compared to the previous situation.

The last step can be omitted when one considers invariant nonlinear systems, like an aircraft performing a rapid manoeuvre in the same region of the flight envelope. However, when the nonlinear system can change suddenly in time, e.g., a damaged aircraft, one should ignore the data before the change, since they are not representative anymore for the actual current nonlinear system. This is the purpose of the last step.

The last remaining topic in this algorithm setup to be discussed is the subset selection stopping criterion. Several criteria can be used for this purpose. Klein and Morelli [16] mention in this respect the predicted sum of squares (PRESS), the predicted square error (PSE), the coefficient of determination $R^{2}$, and the fit error. These metrics have been evaluated in the previous work [5].

In this context, an alternative criterion has been searched for, which still has the same structure of a data fitting and an overfitting penalty term as in the PSE. A good criterion has been found in [24], namely, the Schwarz criterion or Bayesian Information Criterion (BIC) [25], which can be considered as a specific case of the Akaike Information Criterion (AIC):

$$
\mathrm{BIC}_{j}(\alpha)=N \ln \left(\mathrm{MSFE}_{j}\right)+p \ln (N) .
$$

If $\mathrm{BIC}_{j-1}-\mathrm{BIC}_{j}$ remains below a certain criticality threshold $\epsilon_{\mathrm{crit}_{1}}$, the verification serves as a satisfactory subset selection stopping criterion. Implementation of this criterion for online applications has shown to work well. The potential of this routine is shown in the example in the next section.

${ }^{*}$ This indicates a low level of uncertainty. Based upon the data content used until now, an accurate estimate has been obtained. 


\section{APPLICATION TO AN AERODYNAMIC MODEL DATA SET}

This application scenario is inspired by the so-called Bijlmermeer disaster of EL AL flight 1862, where a Boeing 747-200 Cargo aircraft of Israel's national airline EL AL lost two engines immediately after take-off from Amsterdam airport Schiphol in the Netherlands and crashed into an apartment building in the neighborhood while trying to return to the airport. A detailed simulation model of this damaged aircraft is available from the Dutch aerospace laboratory NLR. This RECOVER (REconfigurable COntrol for Vehicle Emergency Relief) benchmark model is discussed in detail by Smaili et al. [26,27] and has been used in the earlier publications of this research project, but also in earlier versions by a number of investigators and organizations [28-30].

The simulation benchmark for evaluating fault tolerant flight controllers as discussed by Smaili et al. [26] contains six benchmark fault scenarios, enumerated in Table 1. These failure cases have varying criticality. Figure 2 shows the failure modes and structural damage configuration of the Flight 1862 accident aircraft, which is the most important fault scenario in the simulation benchmark and which is used in this application section.

Table 1 Garteur FM-AG(16) RECOVER benchmark fault scenarios Smaili [27]

\begin{tabular}{|c|c|c|}
\hline Failure mode & Reconfiguration & Criticality \\
\hline No failure & $\mathrm{N} / \mathrm{A}$ & None \\
\hline \multirow{3}{*}{ Stuck or erroneous elevator } & Stabilizer & \multirow{3}{*}{ Major } \\
\hline & Ailerons (symmetric) & \\
\hline & Differential thrust & \\
\hline \multirow{2}{*}{ Stuck or erroneous aileron } & Ailerons (other) & \multirow{2}{*}{ Major } \\
\hline & Spoilers & \\
\hline \multirow{5}{*}{ Elevator/stabilizer runway } & Elevator & \multirow{5}{*}{ Catastrophic } \\
\hline & Ailerons & \\
\hline & Flaps & \\
\hline & Thrust & \\
\hline & Use of static stability & \\
\hline \multirow{2}{*}{ Stuck, erroneous, or rudder runway } & Remaining surfaces & \multirow{2}{*}{ Catastrophic } \\
\hline & Asymmetric thrust & \\
\hline \multirow{2}{*}{ Loss of vertical tail surface } & Differential thrust & \multirow{2}{*}{ Catastrophic } \\
\hline & Differential speedbrakes & \\
\hline \multirow{3}{*}{ Engine separation and structural damage } & Remaining surfaces & \multirow{3}{*}{ Catastrophic } \\
\hline & Remaining engines & \\
\hline & Remaining sensors & \\
\hline
\end{tabular}




Aircraft systems
Hydraulic Nos. 3 and 4 off
Engines Nos. 1 and 2 thrust asymmetry
Lower rudder lag
Mass properties
Engines Nos. 3 and 4 weight loss, $4014 \mathrm{~kg}$ each
Pylons Nos. 3 and 4 weight loss, $\pm 1000 \mathrm{~kg}$ each
Lateral center of gravity displacement
Total weight loss: $10028 \mathrm{~kg}$
Aerodynamics
Lift loss due to wing damage $\left(\Delta L_{\text {wing }}\right)$
Rolling moment due to wing damage $\left(\Delta L_{\text {wing }}\right)$
Drag due to wing damage $\left(\Delta D_{\text {wing }}\right)$
Yawing moment due to wing damage $\left(\Delta N_{\text {wing }}\right)$
Pitching moment due to wing damage $\left(\Delta M_{\text {wing }}\right)$
Right inboard aileron and spoiler 10 and 11
aerodynamic efficiency loss

Figure 2 Failure modes and structural damage configuration of the Flight 1862 accident aircraft, suffering right wing engine separation, partial loss of hydraulics and change in aerodynamics [27]

The El Al engine separation scenario is an accurate digital flight data recorder (DFDR) validated simulation of Flight 1862, as explained above, where the loss of hydraulics is taken into account.

Before the actual joint structure selection and regression procedure can be applied, the governing dimensionless aerodynamic forces and moments need to be calculated as dependent variables.

\subsection{Creating the Aerodynamic Forces and Moments}

The aerodynamic forces and moments can be calculated online, making use of the estimated states provided by a Kalman Filter. Measurements of specific forces and angular rates can be corrected for their sensor properties, such as the bias, since it is a part of the state estimator output [31]. Figures 3 and 4 show the time histories of the calculated dimensionless forces and moments coefficients together with the corresponding trajectory of the simulated engine separation scenario. These data will be used for the application of AROLS in the subsequent section. The engine separation failure is triggered $50 \mathrm{~s}$ after the simulation started. Note that engine related contributions in forces and moments are not included here. This study focuses exclusively on the aerodynamic effects. 


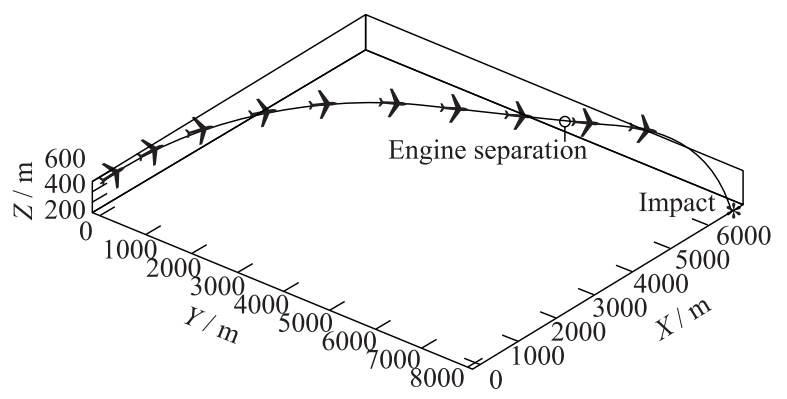

Figure 3 Simulation trajectory of engine separation scenario
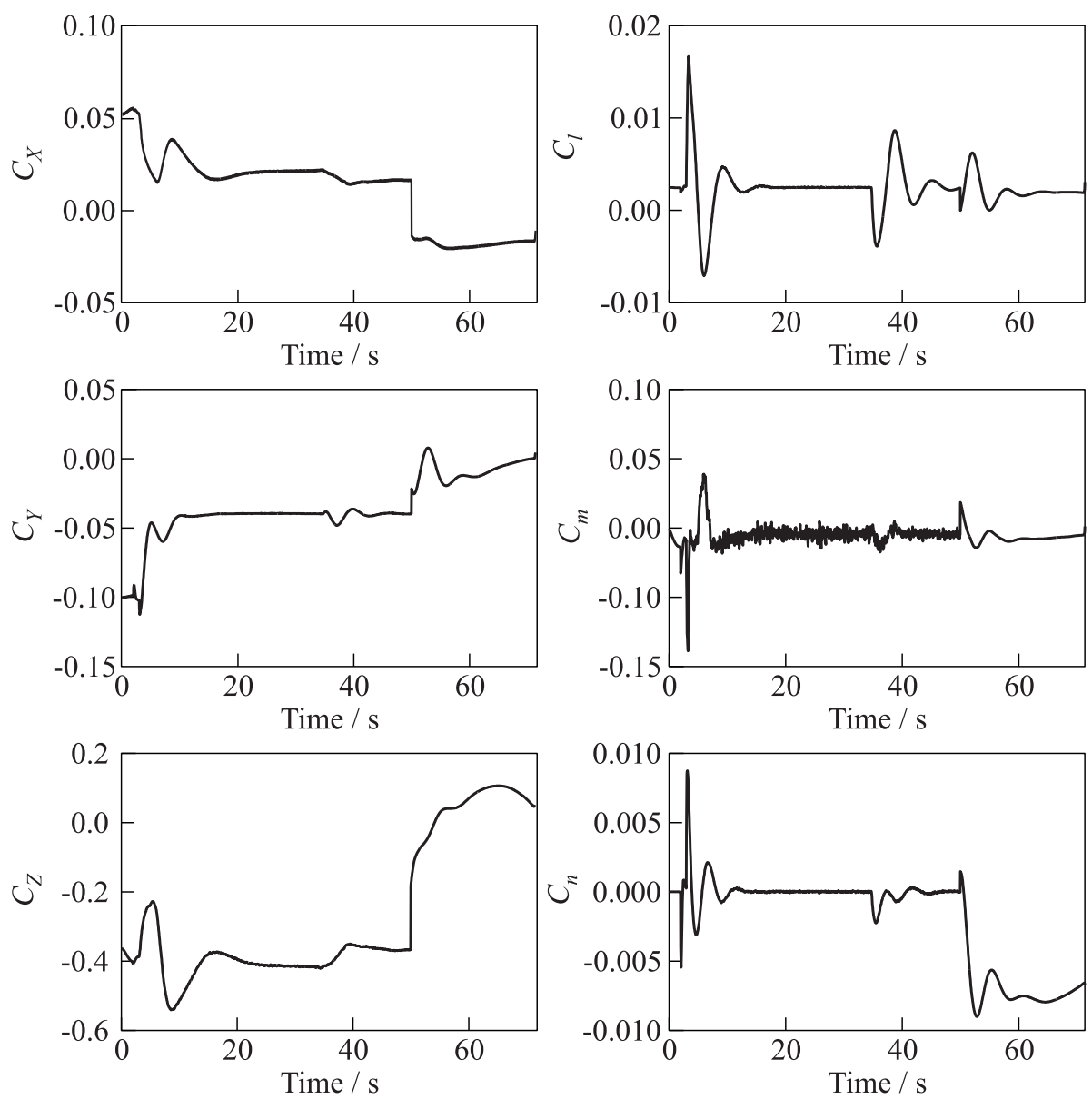

Figure 4 Calculated dimensionless forces and moments coefficients during simulation 


\subsection{Application of Adaptive Recursive Orthogonal Least Squares}

As already mentioned above, the aerodynamic model structure must be selected while the model parameters are being estimated. This is one of the main advantages of the ROLS. Before this model structure selection can be set up, a pool of regressor candidates needs to be defined, i. e., independent variables which are the candidates to be included in the structure.

As an example, the dimensionless moment coefficients $C_{l}$ can be analyzed. In [11], the following independent variables were treated as model regressor candidates. Besides the usual linear independent variables which occur also in the regular aerodynamic models, there are also nonlinear asymmetrical regressor candidates. In case of asymmetric damage, also symmetrical nonlinear regressor candidates need to be taken into account. These three categories contain the following regressors:

- conventional linear independent variables $\beta, p b /(2 V)$, and $r b /(2 V)$ and control surface deflections such as $\delta_{a}$ and $\delta_{r}$;

- nonlinear asymmetrical regressor candidates: $\alpha \beta, \alpha \beta^{2}, \alpha^{2} \beta, \alpha \beta^{3}, \alpha^{2} \beta^{3}$, $\alpha p b /(2 V), \alpha r b /(2 V), \alpha^{2} p b /(2 V), \alpha^{2} r b /(2 V)$, and $\beta^{n}, n=2, \ldots, 5$; and

- symmetrical nonlinear regressor candidates: $1, \alpha, q \bar{c} / V, \alpha^{2}, \alpha^{m}, \alpha q \bar{c} / V$, and $\alpha \delta_{e}, m=3, \ldots, 8$.

Especially in the case of an aircraft with a large set of control surfaces, like Boeing 747, the possibility exists for identical or linear dependent control surface deflections, e.g., $\delta_{e_{o l}}=\delta_{e_{o r}}$ and $\delta_{e_{i l}}=\delta_{e_{i r}}$. However, it is still worthwhile to include them all in the regressor set, since the linear dependency can be lost after a control surface failure. Therefore, the choice has been made to include all of them in the candidate regressor set, but the maximum $v_{m(p)}^{2}(t), p \in \mathcal{P}$, in step 3 of the ROLS algorithm is only selected out of a subset $\mathcal{P}$, consisting of the columns corresponding with the remaining entries in a row reduced echelon form of the upper triangular matrix. In this way, one can avoid collinearities in the identification result.

\subsection{Dimensionless Moment Coefficient $C_{l}$}

Applying the AROLS routine to this stretch of simulation data leads to the results as shown in Fig. 5. For this example, the fit is accurate. Especially after the failure, the real residual illustrates that the structure selection procedure is triggered whenever it deviates significantly from zero.

The parameter estimation results, together with their standard deviations, can be found in Table 2. These results highlight the difference between the situations before and after the failure. Because there is no anomaly before the 

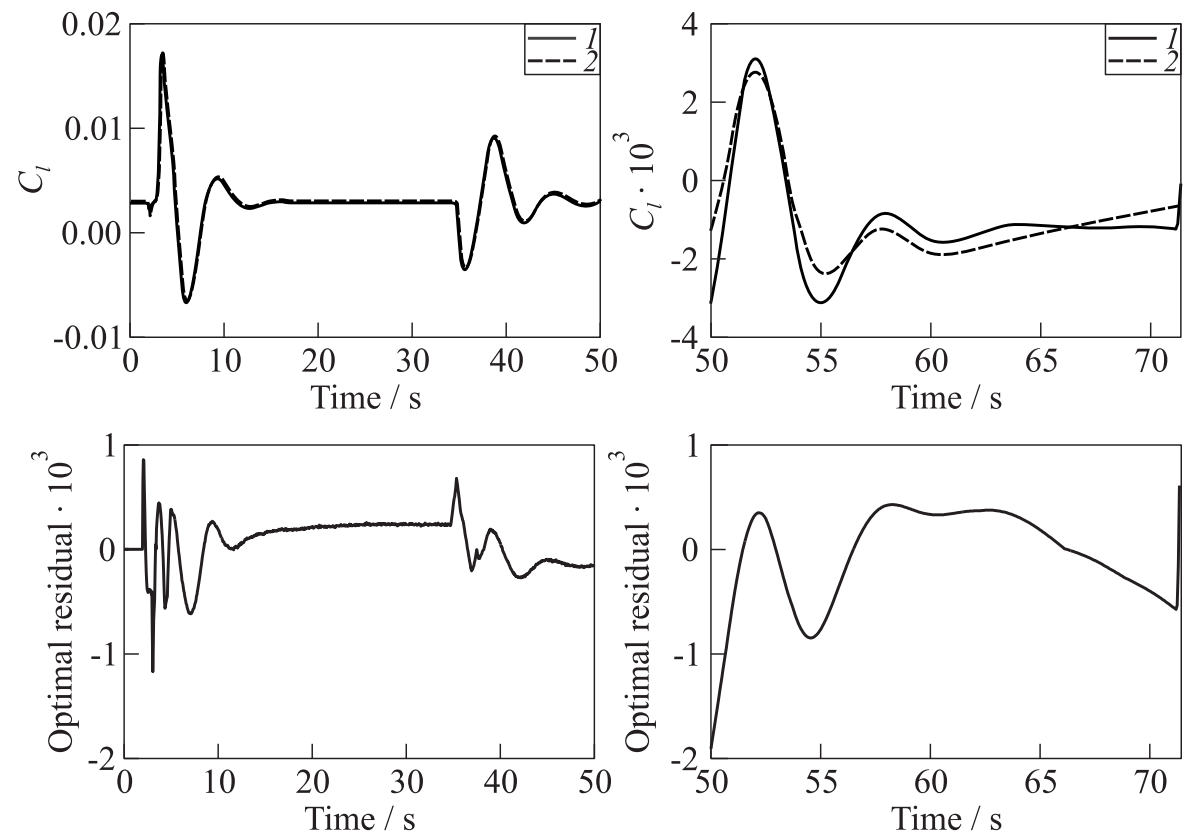

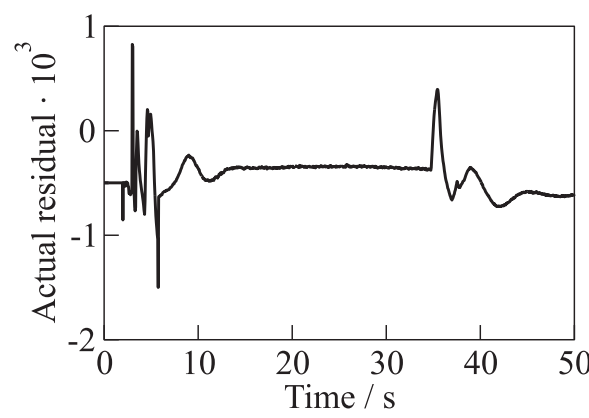

(a)

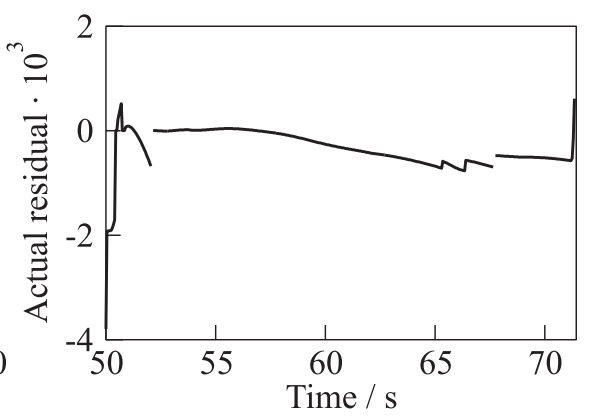

(b)

Figure 5 The AROLS structure selection and parameter estimation results for $C_{l}$ before $(a)$ and after the failure $(b): 1$ - measured; and 2 - reconstructed

failure, the important independent variables for the rolling moment are the conventional ones, namely, the angle of sideslip $\beta$, the roll rate $p b /(2 \mathrm{~V})$, and the ailerons as control effectors. Recall that the inner left aileron $\delta_{a_{I l}}$ is not included, since its deflection is collinear with another aileron surface. This is achieved by considering the row reduced echelon form. However, after the failure, a violent roll-dive manoeuvre follows, as illustrated previously. The influence of the damage on the change in aerodynamics is represented by an additional contri- 
Table 2 The SSPE results for $C_{l}$ for engine separation scenario, before and after failure

\begin{tabular}{ccccc}
\hline \multirow{2}{*}{ Parameter } & \multicolumn{2}{c}{ Prior failure } & \multicolumn{2}{c}{ Post failure } \\
\cline { 2 - 5 } & Estimated & $\sigma$ & Estimated & $\sigma$ \\
\hline$\beta$ & -0.1130 & $1 \%$ & -0.1673 & $1 \%$ \\
$p b /(2 V)$ & -0.3109 & $\approx 0 \%$ & -0.3065 & $1 \%$ \\
$r b /(2 V)$ & - & - & - & - \\
$\delta_{a_{i r}}$ & -0.0005 & $2 \%$ & - & - \\
$\delta_{a_{i l}}$ & - & - & - & - \\
$\delta_{a_{o r}}$ & -0.0004 & $1 \%$ & - & - \\
$\delta_{a_{o l}}$ & 0.0003 & $1 \%$ & - & - \\
1 & - & - & - & - \\
$\alpha$ & - & - & - & - \\
$q \bar{c} / V$ & - & - & -0.4706 & $\approx 0 \%$ \\
All others & - & - & - & - \\
\hline
\end{tabular}

bution from the pitch rate $q \bar{c} / V$, since decoupling of longitudinal and lateral regressors does not hold anymore in this scenario. It should also be noted that the ailerons are not significant regressors after the failure. This is because they cannot move anymore as can be seen in Fig. 2. A few spoilers remain effective, but these are not sufficiently excited by the classical control system in this short time span to allow a successful identification of their individual control efficiencies. Therefore, separate surface excitation (SSE) is needed $[32,33]$. In combination with the SSPE algorithm, this will provide reliable values for the primary control efficiencies. Subsequently, this information can be used by the model-based control algorithm, such as adaptive nonlinear dynamic inversion (ANDI).

The development of the structure selection algorithm is displayed in Figs. 6 and 7 . Figure 6 shows the number of regressors which have been included, and Fig. 7 displays the time history of the monitoring criteria. Between 5 and $10 \mathrm{~s}$, when the dynamic right-hand turn is executed, the rolling moment is excited significantly and the model structure is extended accordingly. Soon after the start of the turn, a repetition of the structure selection phase is triggered and at the end of the turn, the structure is frozen and will not change anymore till the failure occurs. When the engines separate, $\overline{\varepsilon_{M_{s}}^{2}}(t)$ increases dramatically while the maximum standard deviation shows no significant increase. This triggers step 9 in the ROLS algorithm and the identification procedure is reset, ignoring all data collected before since they have become irrelevant for the new configuration. The reinitialization is triggered and after some model development updates only three regressors are retained, namely, $\beta, p b /(2 V)$, and $q \bar{c} / V$ are included, since they represent the most important dynamics which are dom- 


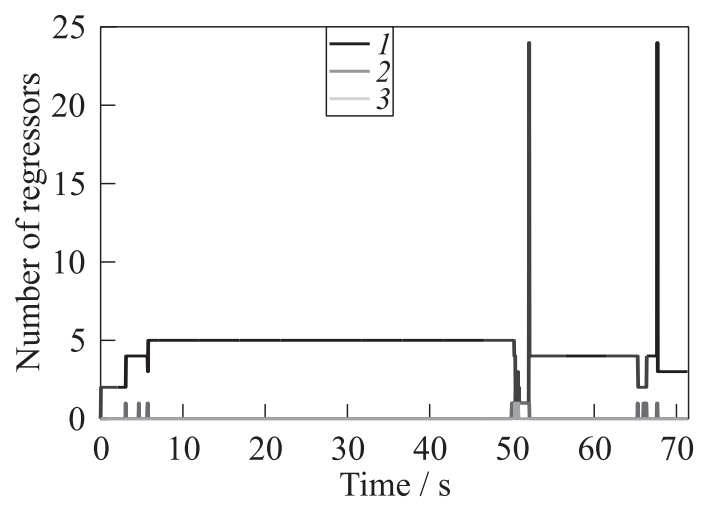

Figure 6 Number of regressors included for $C_{l}(1)$ and unfreezing moments $(2) ; 3-$ all triggers are reset
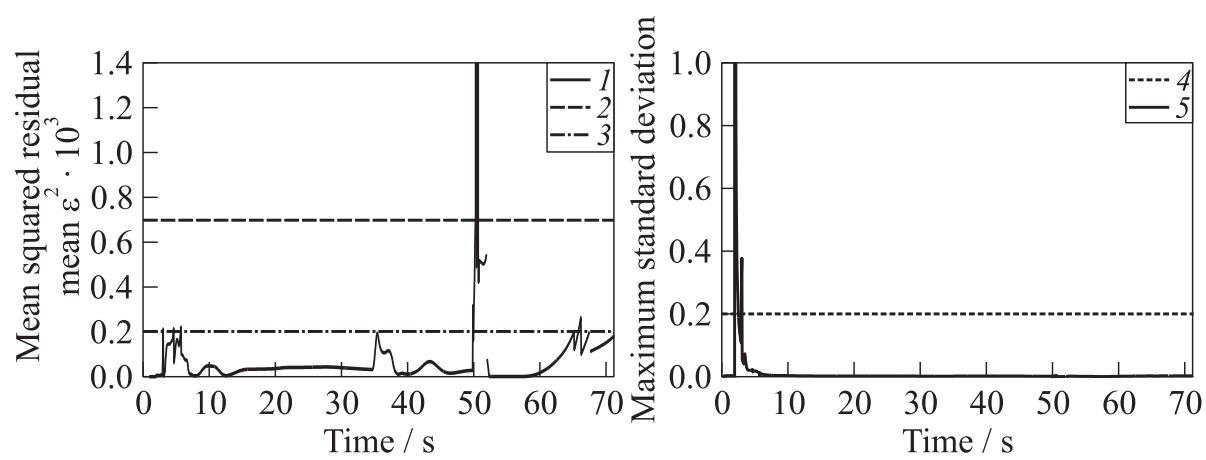

Figure 7 The AROLS structure selection and parameter estimation result after failure for $C_{l}: 1-$ mean squared residual; 2 to $4-$ threshold 1 to 3 ; and $5-$ maximum coefficient standard deviation

inant in the longer term. Initially, also the yaw rate $r b /(2 V)$ is included, but its relevance, together with its coefficient, decreases over time, and the regressor becomes redundant from $68 \mathrm{~s}$ onward. After this, the structure selection procedure freezes again, since $\overline{\varepsilon_{M_{s}}^{2}}(t)$ becomes smaller again, but not far below the threshold $\xi_{M_{s_{1}}}$, pointing out that an optimal fit has not yet been achieved. Towards the end, it can also be seen that the mean squared residual increases again, due to new slower dynamics which become influential. These dynamics would unfreeze the selection once again if the aircraft would not have hit terrain. 


\section{CONCLUDING REMARKS}

Initial simulation results have shown that AROLS is a suitable new algorithm to develop an aerodynamic model structure and to estimate the model parameter values. The structure selection and regression algorithm is made adaptive, so that it is reconfigurable for sudden as well as gradual changes in the aerodynamic model structure as a consequence of aerodynamic damage. Faults are detected successfully and it is even possible to determine the time instant when the fault occurred, even if the fault builds up gradually and is only detected afterwards.

The performance of this algorithm has been evaluated on a high fidelity Boeing 747 simulation model, called RECOVER, which includes a realistic engine separation failure, inspired by and validated by means of the digital flight data recorder readings from El Al Flight 1862 which crashed in Amsterdam in 1992.

This approach works well for changes in the aerodynamic model structure. As a next step, it is interesting to take into account mass property changes as well in the aircraft model, in order to increase the set of failures which can be dealt with by this routine. In this way, a recursive model structure selection and parameter identification algorithm is obtained, which can be used in a subsequent stage for a model-based control algorithm, such as adaptive nonlinear dynamic inversion, in order to achieve fault tolerant flight control.

\section{ACKNOWLEDGMENTS}

The availability of a trustworthy simulation model, including realistic failure scenarios, was a very important contribution to this study. This model, which was developed by Hafid Smaili and Jan Breeman from the Dutch Aerospace Laboratory (NLR), provided an essential instrument in order to test the ability of the two-step method to identify a damaged aircraft model in some realistic failure scenarios. This research was supported by the Dutch Technology Foundation (STW) under project No. 06515.

\section{REFERENCES}

1. Murphy, P., and V. Klein. 2008. Transport aircraft system identification from wind tunnel data. AIAA Atmospheric Flight Mechanics Conference and Exhibit. Honolulu, HI.

2. Song, Y., G. Campa, M. R. Napolitano, B. Seanor, and M. Perhinsch. 2002. Online parameter estimation techniques comparison within a fault tolerant flight control system. AIAA J. Guidance Control Dyn. 25(3):528-37. 
3. Morelli, E., and M. Smith. 2008. Real-time dynamic modeling — data information requirements and flight test results. AIAA Atmospheric Flight Mechanics Conference and Exhibit.

4. Ward, D., J. Monaco, and M. Bodson. 1998. Development and flight testing of a parameter identification algorithm for reconfigurable control. AIAA J. Guidance Control Dyn. 21(6):948-56.

5. Lombaerts, T., Q. Chu, J. Mulder, and D. Joosten. 2007. Real time damaged aircraft model identification for reconfiguring control. AIAA Paper No. 2007-6717.

6. Stroosma, O., H. Smaili, T. Lombaerts, and J. Mulder. 2008. Piloted simulator evaluation of new fault-tolerant flight control algorithms for reconstructed accident scenarios. AIAA Paper No. 2008-6534.

7. Lombaerts, T., H. Huisman, Q. Chu, J. Mulder, and D. Joosten. 2009. Nonlinear reconfiguring flight control based on on-line physical model identification. AIAA J. Guidance Control Dyn. 32(3):727-48.

8. Lombaerts, T., M. Smaili, O. Stroosma, Q. Chu, J. Mulder, and D. Joosten. 2009. Piloted simulator evaluation results of new fault-tolerant flight control algorithm. AIAA J. Guidance Control Dyn. 32(6):1747-65.

9. Edwards, C., T. Lombaerts, and M. Smaili. 2010. Fault tolerant control - a benchmark challenge. Lecture Notes in Control and Information Sciences. Heidelberg: Springer-Verlag. Vol. 399.

10. Draper, N., and H. Smith. 1981. Applied regression analysis. John Wiley \& Sons, Inc.

11. Klein, V., J. Batterson, and P. Murphy. 1981. Determination of airplane model structure from flight data by using modified stepwise regression. Technical Report TP-1916. NASA.

12. Vathsal, E., A. Sarkar, and J. Umakant. 2004. Nonlinear modeling and roll derivatives estimation from flight data using OLS. AIAA Atmospheric Flight Mechanics Conference and Exhibit. Providence, Rhode Island.

13. Morelli, E. 1993. Nonlinear aerodynamic modeling using multivariate orthogonal functions. AIAA Atmospheric Flight Mechanics Conference. Monterey, CA.

14. Morelli, E. 1995. Global nonlinear aerodynamic modeling using multivariate orthogonal functions. J. Aircraft 32(2):270-77.

15. Morelli, E. 1998. Global nonlinear parametric modeling with application to F-16 aerodynamics. American Control Conference. Philadelphia, PA. No. WP04-2. Paper ID i-98010-2.

16. Klein, V., and E. A. Morelli. 2006. Aircraft system identification, theory and practice. AIAA education ser. 141-58.

17. Barron, A.R. 1984. Predicted squared error: A criterion for automatic model selection. Self-organizing methods in modeling. Ed. S. J. Farlow. New York: Marcel Dekker. 54:87-103.

18. Mulder, J. 1986. Design and evaluation of dynamic flight test manoeuvers. Ph.D. Thesis. TU Delft, Faculty of Aerospace Engineering.

19. Luo, W., and S. Billings. 1995. Adaptive model selection and estimation for nonlinear systems using a sliding data window. Signal Proc. 46:179-202. 
20. Luo, W., S. Billings, and K. Tsang. 1996. On-line structure detection and parameter estimation with exponential windowing for nonlinear systems. Eur. J. Control 2:291-304.

21. Fung, C., S. Billings, and W. Luo. 1996. On-line supervised adaptive training using radial basis function networks. Neural Networks 9(9):1597-617.

22. Luo, W., and S. Billings. 1998. Structure selective updating for nonlinear models and radial basis function neural networks. Int. J. Adaptive Control Signal Proc. 12:325-45.

23. Chen, S., S. Billings, and W. Luo. 1989. Orthogonal Least Squares methods and their application to non-linear system identification. Int. J. Control 50(5):1873-96.

24. Miller, A. J. 2002. Subset selection in regression. Chapman \& Hall/CRC.

25. Schwarz, G. 1978. Estimating the dimension of a model. Ann. Stat. 14(3):416-64.

26. Smaili, M., J. Breeman, T. Lombaerts, and D. Joosten. 2006. A simulation benchmark for integrated fault tolerant flight control evaluation. AIAA MST.

27. Smaili, M., J. Breeman, and T. Lombaerts. 2008. A simulation benchmark for aircraft survivability assessment. 26th Congress (International) of the Aeronautical Sciences. Anchorage, AK. No. ICAS 2008-9.3.2.

28. Szaszi, I., S. Ganguli, A. Marcos, G. J. Balas, and J. Bokor. 2002. Application of FDI to a nonlinear Boeing 747 aircraft. 10th Mediterranean Conference on Control and Automation. Lisbon, Portugal.

29. Marcos, A., and G. Balas. 2003. A Boeing 747-100/200 aircraft fault tolerant and fault diagnostic benchmark. Technical Report AEM-UoM-2003-1.

30. Maciejowski, J., and C. Jones. 2003. MPc fault tolerant flight control case study: Flight 1862. IFAC Safeprocess Conference.

31. Lombaerts, T. 2010. Fault tolerant flight control. A physical model approach. Ph.D. Thesis. Delft University of Technology.

32. Hamel, P. G., and R. V. Jategaonkar. 1996. Evolution of flight vehicle system identification. J. Aircraft 33(1):9-28.

33. Weiss, S., H. Friehmelt, E. Plaetschke, and D. Rohlf. 1996. X-31A system identification using single-surface excitation at high angles of attack. J. Aircraft 33(3):48590 . 